\title{
Computational simulation of frontal impact of motorcycle telescopic fork
}

\begin{abstract}
This paper presents modelling aspects of a high fidelity finite element model of a motorcycle telescopic fork. For validation purpose, a series of impact tests of which a frontal impact load imposed on the individual fork by a rigid striker was conducted using factorial experiment approach. The model was then used to simulate all the impacts accordingly. The outcomes were evaluated in terms of permanent deformations of the fork, energy dissipated in the impact, and the velocity and displacement time histories of the striker. The overall performance and sensitivity of the model was also assessed using the factorial analysis. The deviations of time histories data were quantified by mean error, mean absolute error and root mean square error. The key responses of the fork were found to be successfully simulated and compared well to the test results. The computed largest value among the respective metrics over the total errors was $6.2 \%$ of which corresponding to the root mean square error of the displacement time histories data, whereas others were less than $3 \%$. It is thus concluded that the fork model has been successfully validated and the modelling methods has been established. Recommendations for improvements were also given in the paper.
\end{abstract}

Keyword: Finite element modelling; Impact simulation; Motorcycle crashes; Telescopic fork 